\title{
Calculation of Talocrural Joint Axis Motion by Approximating Trochlea Tali with Conical Side Surface
}

\author{
${ }^{1}$ Kenta Nomura, ${ }^{2}$ Shinichi Kosugi, ${ }^{3}$ Yasuhito TANAKA, ${ }^{1}$ Hiroshi Takemura \\ ${ }^{1}$ Department of Mechanical Engineering, Tokyo University of Science, Japan; \\ ${ }^{2}$ Department of Orthopedic Surgery, Nara Prefectural Seiwa Medical Center, Japan; \\ ${ }^{3}$ Department of Orthopedic Surgery, Nara Medical University, Japan; \\ 7515648@ed.tus.ac.jp
}

\begin{abstract}
The motion of the talus, the most complex and important bone in ankle motion, is determined by the geometric characteristics of the articular surface of the talocrural joint, known as the trochlea tali. Therefore, modeling the geometric features of the trochlea tali is important for various fields. The purpose of this study is to approximate the rotation axis of the talocrural joint, which is important in the motion of the ankle foot with a conical model. In this experiment, a foot in four types of postures was photographed using computerized tomography (CT). An approximate cone was generated from point cloud data of the trochlea tali, obtained in this CT imaging experiment. In addition, the relationship between the rotation axis of the talus obtained by this experiment and the approximated cone was confirmed by this study. The results show that the axis of rotation of the talocrural joint moves along the side surface of the approximated cone, formed by two protruded shapes of trochlea tali. This suggests that the proposed method can be used to model the rotation axis of the talocrural joint with the side surface of the cone.
\end{abstract}

Keywords: Trochlea tali; Modeling with infinite cone side surface; Rotation axis of the talocrural joint; Computerized tomography; Globally optimal iterative closest point.

\section{Introduction}

The kinematic properties of the talus, the most complex and important bone in ankle motion, are the result of complex interactions between bone joint morphology, ligament restraint and muscle contraction [1]. However, the basic pattern of movement is determined by the geometric characteristics of the articular surface of the talocrural joint, called the trochlea tali. The modeling of the geometric features of the trochlea tali is extremely important for artificial joint design and surgical applications $[2,3]$. In previous studies although a method of modeling the trochlea tali has been proposed, it has not been evaluated using the motion of rotation axis of the talus and the fitness of shape of the model [4-8]. In many of the previous works, the relationship between the motion of rotation axis and the model were not discussed, despite its importance in explaining the motion of the talocrural joint. Although it is known that the axis of rotation of the talocrural joint 
moves with ankle motion, many studies approximated the axis of rotation of the talocrural joint with one axis such as two spheres, cylinder and cone. The most common model of trochlea tali in anatomy is a conical approximation as shown in Fig. 1 (a) [9]. Fig. 1 (a) shows the front view of the talus. There are two protrusions on the medial and lateral malleolar facet of trochlea tali (see the protrusion in Fig. 1 (a)), and the inclination of the side, upper and lower surface of the cone are determined based on these the shapes of these protrusions. Another general model is the approximation with two cones made from the medial malleolar facet to the sulcus of trochlea tali and the lateral malleolar facet to the sulcus of trochlea tali, respectively, as shown in Fig. 1 (b) [10, 11]. The modeling procedure for approximation with one cone and two cones differs from one another depending on research methodology, such as, extraction from a computerized tomography (CT) image or a targeted X-ray image of the trochlea tali. Conventional methods approximate the center axis of this approximated cone to the rotation axis of the talocrural joint, however the rotation axis of the talocrural joint moves according to ankle motion. Since joint motion is determined by the geometric characteristics of the bone, we assumed that the axis of rotation of the talocrural joint is moving along the side surface of the approximated cone

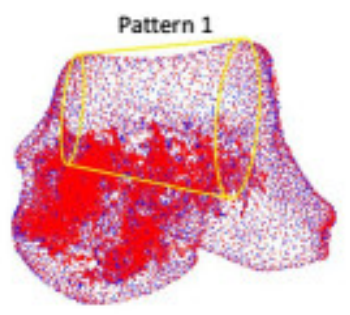

(a) With one cone (Pattern 1)

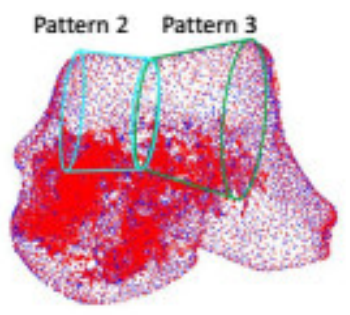

(b) With two cones (Pattern 2 \& 3)

Figure 1 Approximation of trochlea tali with cone

In this study, we propose a method for modeling the trochlea tali with the side surface of an infinite cone and confirm whether the axis of rotation of the talocrural joint can be represented by the proposed model. In this study, three kinds of conical approximation methods, one cone using the medial malleolar facet and the lateral malleolar facet (pattern 1), one cone using the medial malleolar facet to the sulcus of trochlea tali (pattern 2), and another one using the lateral malleolar facet to the sulcus of trochlea tali (pattern 3), are performed by a simple calculation using the extracted point cloud data obtained from CT image data.

\section{Estimation Methods}

In this study, the talar pulley surface is approximated by the side surface of an infinitely long cone without a bottom. An infinitely long cone is uniquely determined by specifying six parameters $\mathrm{A}=(a, b, c, \alpha, b, \vartheta)$. The six parameters are defined by $(a, b, c, \alpha, b, \vartheta)$ in the Fig. 2 (The vertex of the cone $\left.\mathbf{0}=(a, b, c) \in \boldsymbol{R},-\frac{\pi}{2} \leq \alpha \leq \frac{\pi}{2}, 0 \leq \beta<2 \pi, 0 \leq \theta<\frac{\pi}{2}\right)$ through the central axis of the cone is expressed by (1).

$$
\mathbf{n}=[\cos (\alpha) \cos (\beta), \cos (\alpha) \sin (\beta), \sin (\alpha)]
$$


Kenta Nomura, Shinichi Kosugi, Yasuhito Tanaka, Hiroshi Takemura; Calculation of Talocrural Joint Axis Motion by Approximating Trochlea Tali with Conical Side Surface. Journal of Biomedical Engineering and Medical Imaging, Volume 4, No 2, April (2017), pp 1-9

Defining the point group data $P_{i}=\left[x_{i}, y_{i}, z_{i}\right], x, y, z \in R, 1 \leq i \leq N$ ' ( $N$ is the number of points), the distance $r$ from the central axis $\boldsymbol{n}$ to the point cloud $\boldsymbol{P}_{\boldsymbol{i}}$ is expressed by (2).

$$
r=\left|\boldsymbol{O} \boldsymbol{P}_{\boldsymbol{i}}\right| \sin (\gamma)
$$

Where $\boldsymbol{\gamma}$ is the angle between $\boldsymbol{n}$ and $\boldsymbol{P}_{\boldsymbol{i}}$. The distance $\boldsymbol{r}_{\boldsymbol{d}}$ between the point on the approximated conical side surface and $n$ is given by the following equation:

$$
r_{d}=\left|\boldsymbol{O P} \boldsymbol{P}_{\boldsymbol{i}}\right| \cos (\gamma) \tan (\theta)
$$

The solution is when the distance between $r$ and $\boldsymbol{r}_{\boldsymbol{d}}$ is 0 .

$$
\begin{gathered}
\mathrm{J}(\mathrm{A})=\sum_{i=1}^{N}\left(r-r_{d}\right)^{2} \\
J(\overline{\mathrm{A}}) \fallingdotseq \inf \mathrm{J}(\mathrm{A})
\end{gathered}
$$

The six parameters, $\overline{\mathrm{A}}$, that determine the approximate infinitely long cone are calculated by the least squares method. Using the above method, the point cloud data can be approximated on the infinite conical side surface. In this study, only the point cloud data of the protrusions on the medial and lateral malleolar facet of trochlea tali were used for the infinite conical approximation. The upper part of Fig. 3 shows the point cloud data of the talus and the data used for conical approximation. The lower part of Fig. 3 shows the result of the infinite cone approximation using the data from the upper part of Figure 3.

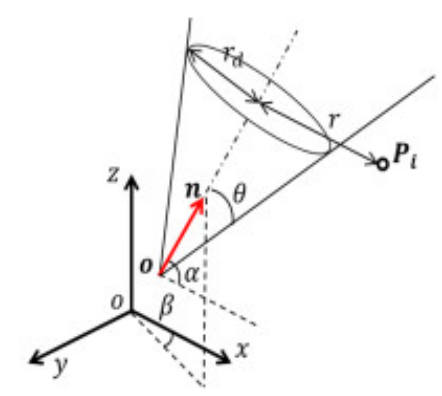

Figure 2 Geometry model of approximation cone
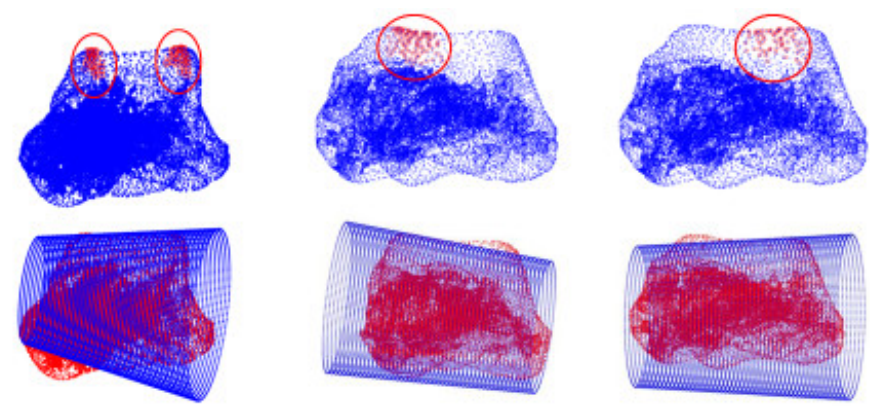

(a) Pattern 1 (b) Pattern 2 (c) Pattern 3

Figure 3 Results of adapting the proposed method to trochlea tali 


\section{Experiment}

\subsection{CT Photography Experiment}

We conducted an experiment using CT to confirm whether the rotation axis expressed by the model of the trochlea tali is the rotation axis of the talus motion. Six healthy adult men, without any disorder or surgical history in the lower limb were recruited and five types of static postures of the right foot with the plantar plate were measured by CT (Aquillion64, Toshiba Medical Systems Co., Ltd.). The age, height, weight, foot length, and foot width were $31.2 \pm 10.2$ years, $169.9 \pm 1.9 \mathrm{~cm}$, $54.6 \pm 2.2 \mathrm{~kg}, 24.2 \pm 0.6 \mathrm{~cm}$, and $9.4 \pm 0.6 \mathrm{~cm}$, respectively. The experiment was approved by the ethics committee of Nara Prefecture Western Medical Center and was conducted in full compliance with the Helsinki Declaration. Informed consent from the subjects was obtained. The plantar plate is made of an acrylic board, so as not to affect the CT photography process. The plantar plate was attached to the subject in order to measure the range of motion (ROM) of foot. In this research, the ROM of foot and the plantar plate were considered equivalent. The ROM of the plantar plate and talus are shown in Table 1. The plantar plates were attached to the foot of the subjects in a left decubitus position and were photographed using CT. An initial posture (IP) (dorsi-plantar flexion (DF-PF), inversion-eversion (IV-EV) and adduction-abduction ( $A D-A B)$ (directions $=0^{\circ}$ ), and four combined postures of DF-PF, IV-EV and $A D-A B$, i.e., $D F+I V+A D, D F+E V+A B, P F+I V+A D$, and $P F$ $+E V+A B$, were photographed. The slice pitch of the $C T$ was $0.5 \mathrm{~mm}$ and the photography range was a cylindrical shape with a diameter of $400 \mathrm{~mm}$ and, in the axial direction, the lower part of the tibia (about $150 \mathrm{~mm}$ from the medial malleolus to the knee side) was taken as the imaging range from the toe. In addition, we instructed all subjects to rest all their fingers on the plantar plate and to maintain foot posture during photography and used a frame, shown in Fig. 4 to support it. In order to avoid excessive radiation exposure, the photography was limited to three types of foot posture for each experiment.. In the first experiment, the initial posture, $D F+I V+A D$, and PF + EV + AB were photographed. In the second experiment, the initial posture, $D F+E V+A B$ and $P F+I V+A D$ were photographed. The interval between the two experiments was approximately one-half year.

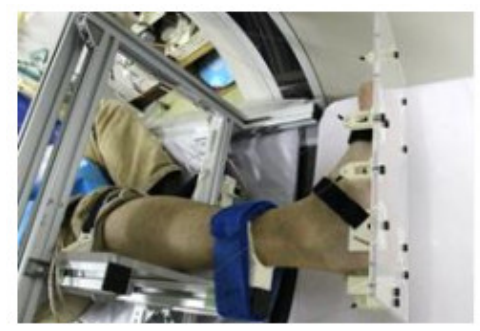

Figure 4 Experimental set-up

Table 1 ROM of plate and talus

\begin{tabular}{lrlrcrl}
\hline \hline & $x[\mathrm{~mm}]$ & $y[\mathrm{~mm}]$ & $z[\mathrm{~mm}]$ & $\theta[\mathrm{deg}]$ & \multicolumn{1}{c}{$\varphi[\mathrm{deg}]$} & \multicolumn{1}{c}{$\psi[\mathrm{deg}]$} \\
\hline plate & $28.7 \pm 5.6$ & $18.9 \pm 2.6$ & $5.7 \pm 3.9$ & $55.3 \pm 6.2$ & $47.8 \pm 13.4$ & $33.6 \pm 7.5$ \\
talus & $1.0 \pm 0.5$ & $16.1 \pm 2.3$ & $3.5 \pm 0.6$ & $47.4 \pm 7.8$ & $3.8 \pm 2.0$ & $10.2 \pm 2.9$ \\
\hline \hline
\end{tabular}


Kenta Nomura, Shinichi Kosugi, Yasuhito Tanaka, Hiroshi Takemura; Calculation of Talocrural Joint Axis Motion by Approximating Trochlea Tali with Conical Side Surface. Journal of Biomedical Engineering and Medical Imaging, Volume 4, No 2, April (2017), pp 1-9

\subsection{Data Analysis}

The data obtained by CT are mostly radiation transmission images. The images are generated in the form of medical image standard called Digital Imaging and COmmunication in Medicine (DICOM). In this experiment, the DICOM and concentration resolution values were $512 \times 512$ pixels and 16 bits, respectively. First, a 3D model of the talus and plate attachment was generated from DICOM using VoTracer (Open source, RIKEN). By arbitrarily setting the CT number range, it is possible to display only specific tissue types, such as bones and soft tissues. In this study, the minimum and maximum CT numbers were set to 100 and 1000, respectively, so that bone areas could be clearly observed in all subjects. The change in posture with 6-DOF of talus and plate attachment from the initial posture in the global coordinate system was calculated. Second, the tibia at the initial posture was defined as the global coordinate system. Third, the change in the posture with 6-DOF of talus and plate attachment from the initial posture in the global coordinate system was calculated after the tibia in the four combined postures were matched with the bone at the initial posture. For the 3D image visualization, the area division software "VoTracer", developed by RIKEN, was used to generate a 3D model of the talus and plate attachment. VoTracer can three-dimensionally extract the area chosen by an operator and output the chosen area in a 3D model format (Stereolithograpy, STL). Region growing, which is one of the functions of VoTracer, was used to extract the area. After extracting the talus and plate attachment area, we obtained the point group data to calculate the change in the bone posture. Fig. 5 shows the definition of the global coordinate system of the tibia. The lateral malleolus, second-toe, and tibial-axis directions from the origin are defined as the $x_{-}, y_{-}$, and $z$-axes of the global coordinate system, respectively. Fig. 5 (a) shows the definition of the origin, $x-$, and yaxis directions. The bottom of the tibia was made to approximate a square. The intersection point of the perpendiculars of two isosceles triangles, whose two extended sides faced each other, was set as the origin. In addition, the perpendicular line of the second-toe direction was defined as the $y$-axis direction, and the axis perpendicular to the $y$-axis direction was defined as the $x$-axis direction. However, in this state, the $x$-axis is around the $y$-axis, the $y$-axis is around the $x$-axis, the $z$-axis has degrees of freedom about both $\mathrm{x}$ and $\mathrm{y}$ axes, and the coordinate system cannot be uniquely determined. Therefore, the rectilinear contour of the tibia is linearly approximated, and the orthogonal coordinate system is uniquely determined by determining the direction of the z-axis, so as to be parallel to the approximated straight line (Fig. 5 (b)). In this manner, the global coordinate system was uniquely determined. Each 3D model of the five types of posture obtained using CT had different positions. Therefore, we need to set a global coordinate to the tibia of each data point when we defined the tibia in a global coordinate system. The four coordinate systems of the tibia in the four combined postures must be matched to the initial posture. In this study, the globally optimal iterative closest point (Go-ICP) algorithm was used for an objective and highly accurate matching of each coordinate system [12, 13]. The Go-ICP algorithm is one of the automated 3D registration methods, which is composed of the branch and bound and voxel ICP and can efficiently derive an optimal solution. By using the Go-ICP algorithm, reliable calculated data can be obtained with high accuracy. In addition, the change in talus and plate attachment posture from the IP was automatically calculated using Go-ICP. The change in talus and plate attachment posture was calculated using a homogeneous transformation matrix, which was used to match the point cloud of 
IP with that of the talus and plate attachment posture that was calculated by Go-ICP. The rotation axis of the talus was calculated by the quaternion from the rotation matrix obtained by Go-ICP.

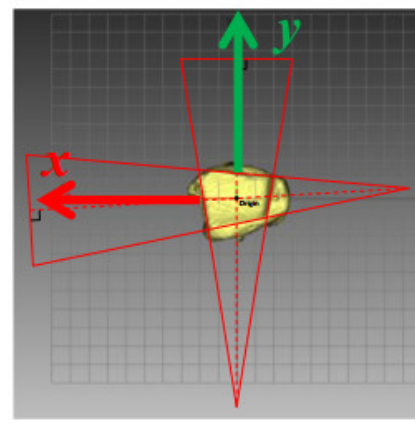

(a) Definition of $x$ and $y$ axis

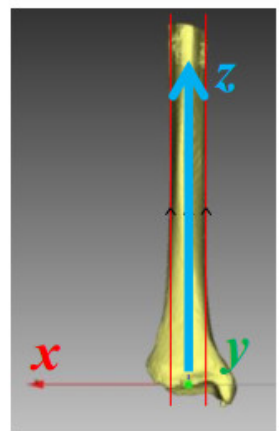

(b) Definition of $z$ axis

Figure 5 Definition of Global coordinate system set on the tibia

\section{Results and Discussion}

\subsection{Results}

In Table 2, the rotation axis of the talus is shown for each motion. The rotation axis in $D F+E V+A B$ to PF + IV + AD is roughly in agreement with the research result of Michael et al., which validates the result [14]. In order to ascertain the assumptions given in section 1, it was evaluated based on the values obtained as follows. First, the difference between the center axis of each approximated cone and the rotation axis of talus was obtained by the inner product of two vectors. Next, the angle $\theta$ of the conical apex of each approximate pattern was subtracted from the difference, and the difference between the conical side surface and the rotation axis was obtained. Fig. 6 shows the RMSE and the standard deviation between the center axis of each approximate cone and the rotation axis of the talus. This graph shows the differences in each approximate pattern in the DF + $I V+A D, D F+E V+A B, P F+I V+A D$ and $P F+E V+A B$ from the left respectively. In addition, the paired t-test was used for confirming the difference in each posture between each approximate pattern. A significant difference appears when the difference in the approximate pattern falls below a level of significance of $5 \%$. In Fig. $6,{ }^{*}$ denotes a pair, where the t-test yielded a value of $p<0.05$.

Table 2 Rotation axis of talus in each motion

\begin{tabular}{crrrr}
\hline \hline & $\begin{array}{c}\text { DF in sagital plane, } \\
\text { relative to transverse } \\
\text { plane (deg) }\end{array}$ & $\begin{array}{c}\text { IV in coronal plane, } \\
\text { relative to transverse } \\
\text { plane (deg) }\end{array}$ & $\begin{array}{c}\text { ER in transverse } \\
\text { plane, relative to } \\
\text { coronal plane (deg) }\end{array}$ & Rotation about (deg) \\
\hline $\mathrm{IP} \rightarrow \mathrm{DF}+\mathrm{IV}+\mathrm{AD}$ & $74.5 \pm 20.6$ & $19.6 \pm 8.4$ & $3.6 \pm 2.4$ & $18.4 \pm 5.5$ \\
$\mathrm{IP} \rightarrow \mathrm{DF}+\mathrm{EV}+\mathrm{AB}$ & $78.8 \pm 6.4$ & $22.4 \pm 9.1$ & $4.4 \pm 3.1$ & $13.2 \pm 6.5$ \\
$\mathrm{IP} \rightarrow \mathrm{PF}+\mathrm{IV}+\mathrm{AD}$ & $53.2 \pm 27.7$ & $6.8 \pm 4.1$ & $6.2 \pm 4.7$ & $27.8 \pm 5.0$ \\
$\mathrm{IP} \rightarrow \mathrm{PF}+\mathrm{EV}+\mathrm{AB}$ & $40.3 \pm 28.8$ & $4.4 \pm 3.4$ & $4.6 \pm 3.5$ & $28.1 \pm 7.7$ \\
$\mathrm{DF}+\mathrm{EV}+\mathrm{AB} \rightarrow$ & $53.4 \pm 27.5$ & $8.7 \pm 5.2$ & $6.0 \pm 4.6$ & $38.5 \pm 8.5$ \\
$\mathrm{PF}+\mathrm{IV}+\mathrm{AD}$ & & & & \\
\hline \hline
\end{tabular}




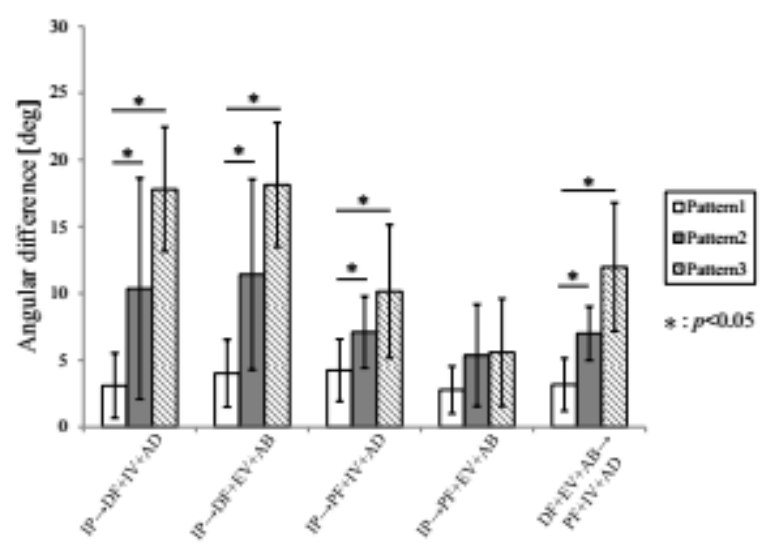

Figure 6 Comparison of angular difference

\subsection{Discussion}

From Table 2, the rotation axis of talus in IP $\rightarrow$ DF and that in IP $\rightarrow$ PF are significantly different. This is consistent with previous studies, indicating that it is inappropriate to approximate the talocrural joint uniaxially. Moreover, the rotation axis of talus in IV + AD and that in EV + AB are only slightly different from one another. This result shows that the rotation axis scarcely changes, since it is constrained by the shape of the trochlea tali, tibia and fibula, unless the movement is performed in the dorsi-plantar flexion direction. From Fig. 6, except for the motion of $P F+E V+A B$, pattern 1 has a smaller difference than the other two patterns. RMSE of pattern 1 also has a smaller difference in PF $+E V+A B$ than in the other two patterns, although no significant difference was obtained in the ttest. This result indicated that the approximation of one cone determined according to two protrusion shapes on the medial and lateral malleolar facet of trochlea tali is the most appropriate while approximating the rotation axis of talocrural joint by the cone side surface. Fig. 7 shows the rotation axis of talus in five kinds of posture and the approximated conical side surface of Pattern 1 (The conical apex is translated to the origin). The blue line represents DF, the red line represents PF, the dotted line represents IV $+A B$, the solid line represents $E V+A D$, and the black line represents $D F$ $+E V+A B \rightarrow P F+I V+A D$ in Fig. 7. This figure shows that the rotation axis in each posture is located near the approximated cone side surface. In addition, from the graph of Fig. 6, the angular difference between the rotation axis and the approximated cone side surface is extremely small at $4.2^{\circ}$ for the maximum in RMSE. These results suggest that the rotational axis can be calculated on the approximated conical side surface. This method may turn out to be useful, because it is possible to capture the rotation axis of the talus having individual shape differences in every talocrural joint motion from an approximated conical side surface using a simple calculation with an accuracy of $4.2^{\circ}$ by RMSE. In addition, the axis of rotation of the talocrural joint is moving along the side surface of the approximated cone determined by the shapes of the two protrusions of the trochlea tali. This knowledge is useful in the field of anatomy, artificial joint design, and surgical applications. 


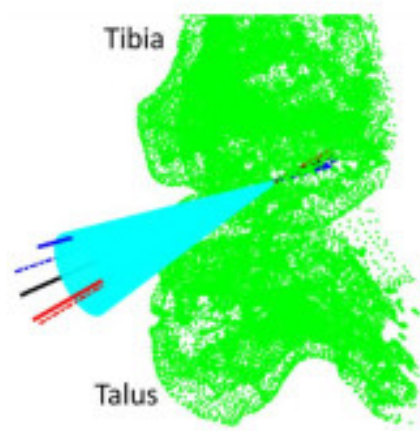

Figure 7 Approximated cone side surface and rotation axis of talus during each motion

\subsection{Limitations}

There are a few limitations to this study. We could not confirm whether the data generated for proof that cone model is useful or sufficient (six subjects and four types of posture). The number of subjects and postures can be increased to obtain better reliability. Moreover, it is unknown whether this method can be applied to the talus of individuals apart from healthy adult men, e.g., the talus of women or children, an artificial talus, or the talus of arthrosis patients, because all of the subjects considered were healthy adult men. However, since the rotation axis movement depended on the shape of the trochlea tali, it is expected that the difference due to gender or age is not large. In the future, it is desirable to increase the number of subjects and to verify whether this method is applicable to the talus of any person.

\section{Conclusion}

Our purpose of the study is to approximate the rotation axis of the talocrural joint, which is important in the motion of the ankle by a conical model. We assumed that the rotation axis of the talus is moving along the side surface of the approximated cone. It is confirmed by three approximation methods with the cone obtained by point cloud data of the talus and experiments using CT photography. The results show that the axis of rotation of the talocrural joint is moving along the side surface of the approximated cone, which is formed by two protrusions of the trochlea tali. This result suggests that the proposed method can model the rotation axis of the talocrural joint with the side surface of the cone. Furthermore, the rotation axis of the talus moves along the side surface of the approximated cone, which is obtained by two protrusions in the medial malleolar facet and the lateral malleolar facet of trochlea tali. This knowledge is expected to be applied in various fields such as anatomy, artificial joint design, surgical operation, and biomechanics.

\section{REFERENCES}

[1] Schunke, M. and E. Schulte, Thieme atlas of anatomy : General anatomy and musculoskeletal system :Second edition, IGAKU-SHOIN, 2011.

[2] Chou, L.B., et al., Osteoarthritis of the ankle: the role of arthroplasty. The Journal of the American Academy of Orthopaedic Surgeons. 2008. 16(5): p. 249-259. 
Kenta Nomura, Shinichi Kosugi, Yasuhito Tanaka, Hiroshi Takemura; Calculation of Talocrural Joint Axis Motion by Approximating Trochlea Tali with Conical Side Surface. Journal of Biomedical Engineering and Medical Imaging, Volume 4, No 2, April (2017), pp 1-9

[3] Leardini, A., et al., Mobility of the human ankle and the design of total ankle replacement. Clinical Orthopaedics and Related Research. 2004. 424: p. 39-46.

[4] Parr, W.C., et al., Calculating the Axes of Rotation for the Subtalar and Talocrural Joints Using 3D Bone Reconstructions. Journal of Biomechanics. 2012. 45(6): p. 1103-1107.

[5] Inman, V. T., The Joints of the Ankle, Baltimore: Williams \& Wilkins, 1976.

[6] Kempson, G. E., et al., Engineering considerations in the design of an ankle joint. Bio-medical Engineering. 1975. 10(5): p. 166-71.

[7] Pappas, M., et al., Cylindrical total ankle joint replacement. Clinical Orthopaedics and Related Research. 1976. 118: p. 82-92.

[8] Medley, J.B., et al. Surface geometry of the human ankle joint. Engineering in Medicine. 1983. 12(1): p. 35-41.

[9] Stiehl, J.B., Inman's joints of the ankle. 2nd ed., Williams \& Wilkins, Baltimore, 1991.

[10] Siegler, S., et al., The clinical biomechanics award 2013-presented by the international society of biomechanics: new observations on the morphology of the talar dome and its relationship to ankle kinematics. Clinical Biomechanics. 2014. 29(1): p. 1-6.

[11] Barnett, C. H. and Napier, J. R., The axis of rotation at the ankle joint in man. Its influence upon the form of the talus and the mobility of the fibula. Anatomy. 1952. 86: p. 1-9.

[12] Yang, J., et al., Go-ICP: Solving 3D Registration efficiently and globally optimally. International Joint Conference on Computer Vision, Imaging and Computer Graphics Theory and Applications. 2013. :p. 1457-1464.

[13] Yang, J., et al., Go-ICP: A Globally optimal solution to 3D ICP point-set registration. IEEE Transactions on Pattern Analysis and Machine Intelligence. 2016. 38(11): p. 2241 - 2254.

[14] Fassbind, M.J., et al., Evaluating foot kinematics using magnetic resonance imaging: from maximum plantar flexion, inversion, and internal rotation to maximum dorsiflexion, eversion, and external rotation. Journal of Biomechanical Engineering. 2011. 133(10) 